\title{
PRODUÇÃO DE MUDAS CLONAIS DE EUCALIPTO EM ESPUMA FENÓLICA: CRESCIMENTO INICIAL E MORTALIDADE
}

\author{
Paulo Henrique Muller da Silva ${ }^{1}$, Daniel Kager ${ }^{2}$, José Leonardo de Moraes Gonçalves ${ }^{3}$, Antonio Natal Gonçalves ${ }^{4}$
}

(recebido: 23 de março de 2010; aceito: 29 de junho de 2012)

\begin{abstract}
RESUMO: A produção de mudas é um dos pontos cruciais nos empreendimentos florestais e diversos trabalhos procuraram identificar o método mais adequado de produção. Neste estudo, avaliou-se o uso da espuma fenólica na substituição de recipientes e de substrato na produção de mudas clonais de eucalipto. Foram implantados quatro experimentos, sendo dois de viveiro (experimentos 1 e 2) e dois de campo (experimentos 3 e 4) em locais e períodos distintos. Todos os experimentos foram compostos por quatro tratamentos: 1 - tubete de $55 \mathrm{~cm}^{3}$ + substrato comercial; 2 - espuma pequena $\left(60 \mathrm{~cm}^{3}\right) ; 3$ - espuma média $\left(75 \mathrm{~cm}^{3}\right)$; e 4 - espuma grande $\left(90 \mathrm{~cm}^{3}\right)$. Nos experimentos, avaliouse a mortalidade das mudas e a biomassa do sistema radicular e da parte aérea. No experimento 1 , as mudas produzidas nas espumas pequenas obtiveram menor índice de mortalidade (1\%), enquanto no sistema convencional foi de $15 \%$ e observou-se superioridade na produção de biomassa aérea das mudas produzidas na espuma grande. No experimento 2, as mudas produzidas na espuma apresentaram maior sensibilidade ao excesso de água durante a fase de enraizamento, sendo a mortalidade de $40 \%$ para os tratamentos com a utilização da espuma que foi superior ao tratamento convencional (16\%). No experimento 3, conduzido no campo, não foi observada mortalidade das mudas ou diferenças na produção de biomassa entre os tratamentos. No experimento 4, observou-se maior resistência ao déficit hídrico nos tratamentos com as espumas média e grande e maior produção de biomassa aérea no tratamento com a espuma grande.
\end{abstract}

Palavras-chave: Recipiente degradável, Eucalyptus, biomassa aérea e radicular.

\section{USE OF PHENOLIC FOAM TO GROW EUCALYPT CLONAL SEEDLINGS: INITIAL GROWTH AND MORTALITY}

\begin{abstract}
Seedling production is one of the crucial points in the forest companies and studies have been carried out to identify the most appropriate method of production. The utilization of phenolic foam in substitution of plastic tube and organic substrate to grow eucalypt clonal cuttings was evaluated. Four experiments were established, two experiments were set up in nurseries (experiments 1 and 2) and two in the field (experiments 3 and 4). All experiments were composed by four treatments: 1 - conventional system - plastic container of $55 \mathrm{~cm}^{3}$ with commercial substrate (pine bark, vermiculite and peat), 2 - small foam $\left(60 \mathrm{~cm}^{3}\right), 3-$ medium foam $\left(75 \mathrm{~cm}^{3}\right)$ and 4 - large foam $\left(90 \mathrm{~cm}^{3}\right)$. In all experiments the mortality and the root and shot biomass production were evaluated. In the experiment 1, the plants in the small foam presented lower mortality rate $(1 \%)$ and the conventional system showed the highest mortality rate (15\%) and the large foam showed highest value of the shoot biomass. In experiment 2, the mortality rate was $40 \%$ for the foam treatments with higher values when compared to the conventional treatment (16\%). In the experiment 3, field experiment, was not observed tree mortality or differences in biomass production among the treatments. In the experiment 4, was observed better tree response to water stress of the stocks in foams, probably because their larger volume. The plants in medium and larger foams showed $3 \%$ of mortality, while the conventional system had $25 \%$ and the shoot biomass production was highest in the larger foam treatment.
\end{abstract}

Key words: Degradable container, Eucalyptus, shoot and root biomass.

\section{INTRODUÇÃO}

Para o abastecimento do mercado florestal composto por indústrias de base florestal e pequenos consumidores é necessário o plantio de grande quantidade de mudas. Segundo a Associação Brasileira de Produtores de Florestas Plantadas - ABRAF (2009), a área plantada com florestas comerciais em 2008 foi de 640 mil hectares e para atender a essa demanda, estima-se que foram produzidas mais de 1 bilhão de mudas.

\footnotetext{
${ }^{1}$ Engenheiro Florestal, Doutor em Recursos Florestais - Instituto de Pesquisas e Estudos Florestais/IPEF - Avenida Pádua Dias, 11 - Cx. P. 530 - 13400-970 - Piracicaba, SP, Brasil - paulohenrique@ipef.br

${ }^{2}$ Engenheiro Agrônomo, Mestrando em Recursos Florestais - Universidade de São Paulo/USP - Escola Superior de Agricultura "Luiz de Queiroz"/ESALQ - Departamento de Ciências Florestais - Av. Pádua Dias, nº 11 - Agronomia - Cx. P. 09 - Piracicaba, SP, Brasil 13418-900 - daniel.kager@usp.br

${ }^{3}$ Engenheiro Agrônomo, Professor Titular, Doutor em Solos e Nutrição de Plantas - Universidade de São Paulo/USP - Escola Superior de Agricultura "Luiz de Queiroz"/ESALQ - Departamento de Ciências Florestais - Av. Pádua Dias, n 11 - Agronomia - Cx. P. 09 Piracicaba, SP, Brasil - 13418-900 - jlgonca@usp.br

${ }^{4}$ Engenheiro Agrônomo, Professor Doutor em Solos e Nutrição de Plantas - Universidade de São Paulo/USP - Escola Superior de Agricultura "Luiz de Queiroz"/ESALQ - Departamento de Ciências Florestais - Av. Pádua Dias, nº 11 - Agronomia - Cx. P. 09 Piracicaba, SP, Brasil - 13418-900 - natalgon@usp.br
} 
A produção de mudas de espécies florestais em grande escala sempre despertou grandes interesses de instituições de pesquisa, empresas florestais e produtores, levando ao desenvolvimento de diversos trabalhos, visando à melhoria do processo. Em sua maioria, os trabalhos procuraram identificar o método mais adequado de produção de mudas, o recipiente, substrato e a nutrição mais equilibrada para cada uma das fases de desenvolvimento das mudas no viveiro.

No aprimoramento das técnicas de produção florestal, ocorreram diversas modificações no processo produtivo ao longo do tempo (STAPE et al., 2001), onde os viveiros florestais passaram por grande modificações. Houve progressos na qualidade fisiológica das mudas, nas condições de trabalho dos funcionários, no consumo de insumos e nos rendimentos operacionais.

Há anos no Brasil, o sistema mais utilizado para produção de mudas de eucalipto é composto por tubete plástico e substrato orgânico e as mudas são propagadas vegetativamente, em razão dos ganhos obtidos em produtividade e homogeneidade (MCNABB et al., 2002; LINDGREN, 2002). No entanto, ainda existe o interesse em sistemas que substituam o tubete de plástico por materiais degradáveis e/ou estáveis que se incorporam ao solo o que facilitaria o plantio, eliminando algumas operações, e reduziria o risco de contaminar o viveiro com organismos patogênicos com a devolução dos recipientes que podem não ter sido adequadamente limpos no processo de assepsia. Outro ponto importante é que podem ocorrer deformações radiculares causadas por recipientes de paredes rígidas, essas deformaçõos tendem a persistir após a fase de viveiro (FREITAS et al., 2005).

A espuma fenólica, lançada há alguns anos comercialmente para culturas não florestais, é uma opção com potencial para a produção de mudas de eucalipto. A espuma é constituída de um material estéril que não interfere na nutrição e que tem capacidade de prover boa sustentação para a muda, além de ter alta capacidade de retenção de umidade e aeração, características que podem ser modificadas de acordo com a necessidade. Deve se ressaltar que modificações nas dimensões do recipiente e na disponibilidade hídrica devem ser estudas no viveiro e no campo, pois influenciam diretamente no crescimento das mudas durante a fase de viveiro (PRIETO-RUIZ et al., 2007) e podem causar efeitos que persistem após a fase de produção no viveiro (FREITAS et al., 2005). Para algumas culturas agrícolas, a espuma já foi estudada e observou-se comportamento similar ao desenvolvimento das plantas em relação ao procedimento adotado usualmente, em tubete (FERNANDES et al., 2004; MATIAS et al., 1999; PAULUS et al., 2005).

Existem diversos materiais que estão sendo estudados como novas opções de recipientes, preferencialmente biodegradáveis, para a produção de mudas clonais de eucalipto, porém ainda não publicados. Não existem trabalhos na literatura com a utilização da espuma fenólica na produção de mudas clonais de eucalipto.

No presente estudo, objetivou-se avaliar a mortalidade e o crescimento inicial de mudas clonais de eucalipto produzidas em espuma fenólica, visando à substituição do tubete e do substrato pela espuma fenólica no sistema produtivo.

\section{MATERIAL E MÉTODOS}

Foram implantados quatro experimentos, sendo dois experimentos de viveiro e dois experimentos de campo. Em todos os experimentos foram estudados quatro tratamentos, sendo 1 - Sistema convencional (Tubete de $55 \mathrm{~cm}^{3}$ contendo substrato comercial à base de casca de pinus, vermiculita e turfa); 2- Espuma fenólica pequena com 60 $\mathrm{cm}^{3}$ (3,7 x 3,7 x 4,4 cm); 3- Espuma fenólica média com 75 $\mathrm{cm}^{3}(3,7$ x 3,7 x 5,5 cm); e 4- Espuma fenólica grande com $90 \mathrm{~cm}^{3}(3,7 \times 3,7 \times 6,6 \mathrm{~cm})$.

\subsection{Experimentos de viveiro}

\subsubsection{Espécies utilizadas}

Foram utilizadas miniestacas de um híbrido Eucalyptus urophylla com E. resinífera no experimento $1 \mathrm{e}$ de um hibrido E. urophylla com E. grandis no experimento 2 .

\subsubsection{Localização e período de realização dos experimentos}

$\mathrm{O}$ experimento 1 foi conduzido no viveiro do Departamento de Ciências Florestais da ESALQ/USP, localizado no município de Piracicaba (LA $22^{\circ} 70^{\prime}$; LO $47^{\circ}$ 63 '; altitude $490 \mathrm{~m}$; temperatura média $21,6^{\circ} \mathrm{C}$; e precipitação anual média de $1230 \mathrm{~mm}$, no período de janeiro a abril de 2008, durante o verão e outono.

O experimento 2 foi conduzido no viveiro da empresa Lwarcel, localizado no município de Lençóis Paulista (LA 22 32'; LO 48 54'; altitude 560 m; temperatura média $21,8^{\circ} \mathrm{C}$; e precipitação anual média de $1314 \mathrm{~mm}$ ), no período de maio a agosto de 2008 , durante o outono e inverno.

Em ambos os casos, utilizou-se o procedimento padrão dos viveiros, no que se refere à fertilização e à

Cerne, Lavras, v. 18, n. 4, p. 639-649, out./dez. 2012 
irrigação das diferentes fases de produção das mudas. Para as mudas produzidas em espuma, utilizaram-se bandejas diferentes do sistema convencional, em razão das dimensões das espumas e por dispensarem a utilização de recipiente individual (Figura 1).

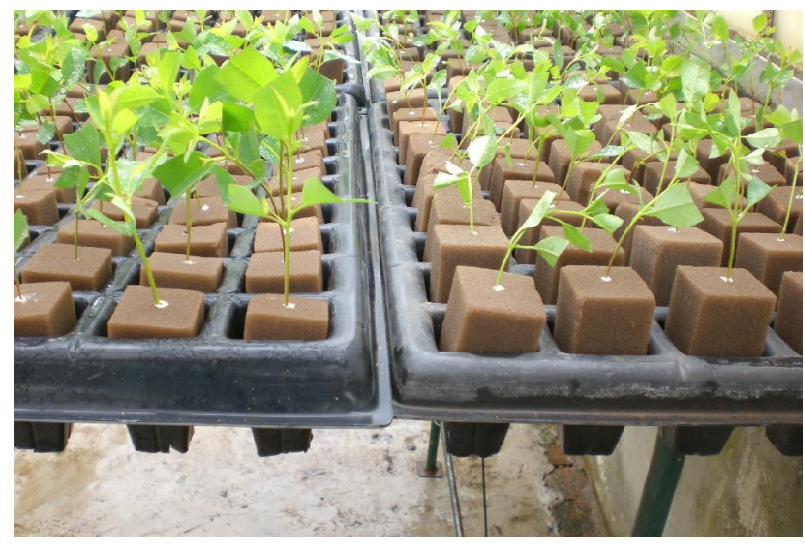

Figura 1 - Tratamentos com a espuma pequena e grande dentro da casa de vegetação.

Figure 1 - Treatments with small and large foam inside the greenhouse.

\subsubsection{Delineamento experimental}

Os experimentos foram montados em blocos ao acaso, com quatro tratamentos e três repetições, sendo que foram utilizadas 60 mudas por repetição.

\subsubsection{Avaliações de mortalidade e crescimento}

As avaliações de mortalidade foram realizadas no termino de cada fase das mudas no viveiro (casa de vegetação: aproximadamente 22 dias; casa de sombra: 5 a 7 dias; e crescimento e rustificação: 55 a 65 dias). A mensuração da biomassa seca da parte aérea e do sistema radicular foram realizadas em 9 mudas de cada tratamento, selecionadas ao acaso na época de expedição para o campo, sendo a idade de 85 dias para o experimento 1 e 95 dias para o experimento 2 .

\subsection{Experimentos de campo}

\subsubsection{Espécies utilizadas}

Foram utilizadas as mudas formadas nos experimentos realizados no viveiro, sendo o híbrido Eucalyptus urophylla com E. resinífera no experimento 3 e o hibrido E. urophylla com E. grandis no experimento 4.
2.2.2 Localização e período de realização dos experimentos

O experimento 3 foi implantado em abril de 2008, durante o outono, na Estação Experimental de Ciências Florestais de Itatinga localizada no município de Itatinga (LA 23 96'; LO 48 86'; altitude 840 m; temperatura média $19,7^{\circ} \mathrm{C}$; e precipitação anual média de $\left.1370 \mathrm{~mm}\right)$.

O experimento 4 foi implantado em setembro de 2008, início da primavera, pela empresa Lwarcel em fazenda localizada no município de Borebi (LA 22 $34^{\circ}$; LO 48 58'; altitude $590 \mathrm{~m}$; temperatura média $21,6^{\circ} \mathrm{C}$; e precipitação anual média de $1355 \mathrm{~mm}$ ).

As atividades operacionais pré-plantio para instalação dos experimentos 3 e 4 foram a aplicação de herbicida na área total, preparo do solo (subsolagem a 50 $\mathrm{cm}$ ) e combate a formigas cortadeiras. O plantio foi realizado de maneira manual e após o plantio foi realizada fertilização.

A diferença na implantação entre os experimentos de campo foi no processo de irrigação, sendo que no experimento 3 foram realizadas duas aplicações com dois litros por mudas (uma logo após o plantio e a outra após uma semana) e, no experimento 4 , foi aplicado $1,2 \mathrm{~g}$ por planta de gel hidratado misturado com o solo junto ao plantio e não foram realizadas irrigações.

\subsubsection{Delineamento experimental}

Os experimentos 3 e 4 foram implantados em blocos ao acaso com quatro tratamentos, 25 e 30 repetições, respectivamente, considerando parcela de uma planta (single tree plot).

\subsubsection{Avaliações de mortalidade e crescimento}

A avaliação da mortalidade ocorreu 30 dias após o plantio, sendo realizado o replantio quando necessário. Foi avaliada a produção de biomassa seca da parte aérea e do sistema radicular das mudas com 3 meses de idade. Foram coletadas 6 plantas, selecionadas aleatoriamente, por tratamento em cada um dos experimentos. As plantas foram coletadas inteiras e secas em estufa de ventilação forçada até atingirem peso constante.

\subsection{Análise estatística}

As variáveis medidas foram submetidas à análise de variância (ANOVA) com nível de significância de 5\%. Foram realizadas análises de variância agrupando os experimentos 1 e 2 e os experimentos 3 e 4 e para cada experimento separadamente. Para as análises nas quais mostraram teste $\mathrm{F}$ significativo foram submetidas a 
comparações múltiplas pelo teste de Tukey (5\%). As análises foram realizadas com o pacote estatístico SAS 9.1.

\section{RESULTADOS E DISCUSSÃO}

As análises mostraram que os experimentos não devem ser agrupados, pois existe maior variação entre eles do que entre os tratamentos avaliados (Tabela 1).

Verificou-se nas análises individuais dos experimentos respostas distintas em relação às características avaliadas. No experimento 1, foram observadas diferenças significativas entre os tratamentos $(\mathrm{p}<0,05)$ na mortalidade das mudas na fase de expedição e na biomassa aérea. No experimento 2, as características com diferenças entre os tratamentos foram mortalidade e relação parte aérea/sistema radicular. No experimento 3, não foram observadas diferenças significativas entres os tratamentos e no experimento 4 observou-se diferenças na mortalidade e na produção de biomassa aérea entre os tratamentos (Tabela 2).

\subsection{Mortalidade das mudas nos experimentos de viveiro}

Os resultados de mortalidade durante a produção das mudas foram distintos entre experimentos. No experimento 1 , observou-se que o tratamento com a espuma pequena apresentou menor mortalidade, enquanto que as mudas produzidas em espuma grande apresentaram mortalidade equivalente ao tratamento convencional no momento da expedição das mudas. No entanto, ocorreram taxas

Tabela 1 - Análise de variância agrupando os experimentos em viveiro (experimentos 1 e 2) e campo (experimentos 3 e 4 ).

Table 1 - Variance analysis by grouping of nursery (experiments 1 and 2) and field (experiments 3 and 4) experiments.

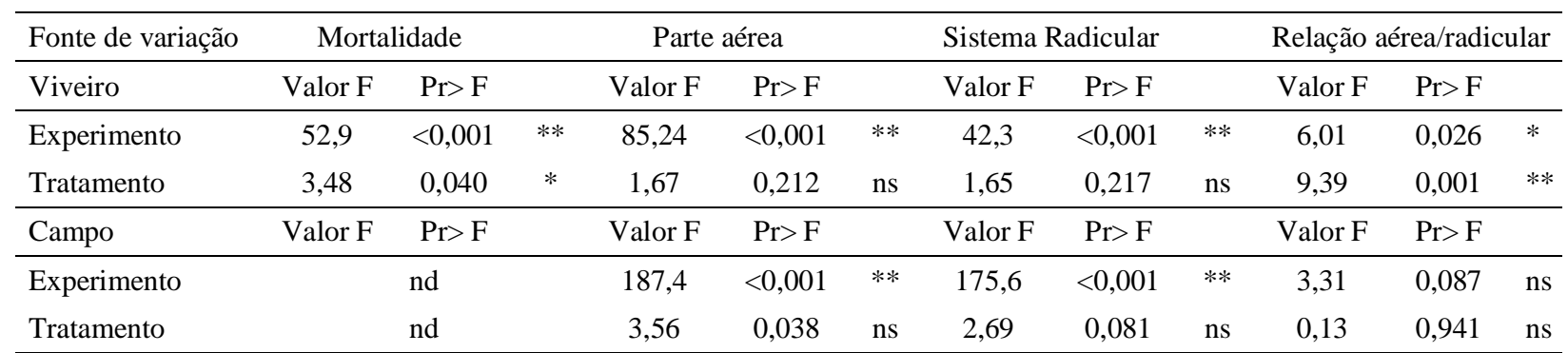

Legenda: $\operatorname{Pr}>\mathrm{F}=$ probabilidade $\mathrm{F} ; \mathrm{ns}=$ não significativo; $*$ significativo a $5 \%$; $* *$ significativo a $1 \%$; nd = não definido

Tabela 2 - Análise de variância dos quatro experimentos separados.

Table 2 - Variance analysis of the four experiments separated.

\begin{tabular}{|c|c|c|c|c|c|c|c|c|}
\hline \multirow{2}{*}{ Características } & \multicolumn{4}{|c|}{ Experimento 1} & \multicolumn{4}{|c|}{ Experimento 2} \\
\hline & Valor de F & $\operatorname{Pr}>\mathrm{F}$ & & DMS & Valor de F & $\operatorname{Pr}>\mathrm{F}$ & & DMS \\
\hline Mortalidade na expedição & 11,3 & 0,003 & $* *$ & $3,9 \%$ & 6,53 & 0,01 & $*$ & $8,0 \%$ \\
\hline Biomassa parte aérea (pa) & 5,70 & 0,04 & $*$ & $0,28 \mathrm{~g}$ & 4,04 & 0,51 & ns & 0,24 \\
\hline Biomassa radicular (r) & 1,19 & 0,37 & ns & $0,34 \mathrm{~g}$ & 1,97 & 0,19 & $\mathrm{~ns}$ & 0,07 \\
\hline \multirow[t]{3}{*}{ Relação pa/r } & 2,93 & 0,10 & ns & 1,85 & 7,2 & 0,01 & $*$ & 1,7 \\
\hline & \multicolumn{4}{|c|}{ Experimento 3} & \multicolumn{4}{|c|}{ Experimento 4} \\
\hline & Valor de F & $\operatorname{Pr}>\mathrm{F}$ & & DMS & Valor de F & $\operatorname{Pr}>F$ & & DMS \\
\hline Mortalidade & nd & $\mathrm{Nd}$ & nd & $\mathrm{Nd}$ & 2,91 & 0,03 & $*$ & $2,6 \%$ \\
\hline Biomassa parte aérea $(\mathrm{pa})$ & 0,74 & 0,55 & ns & $2,97 \mathrm{~g}$ & 6,23 & 0,02 & $*$ & $56,5 \mathrm{~g}$ \\
\hline Biomassa radicular (r) & 0,71 & 0,57 & ns & $0,69 \mathrm{~g}$ & 2,7 & 0,11 & ns & $18,5 \mathrm{~g}$ \\
\hline Relação pa/r & 0,01 & 0,99 & $\mathrm{~ns}$ & 2,25 & 0,25 & 0,85 & ns & 3,3 \\
\hline
\end{tabular}

Legenda: $\operatorname{Pr}>\mathrm{F}=$ probabilidade $\mathrm{F} ; \mathrm{DMS}=$ diferença mínima significativa; ns = não significativo; $*$ significativo a $5 \% ; * *=$ significativo a $1 \%$; nd = não definido

Cerne, Lavras, v. 18, n. 4, p. 639-649, out./dez. 2012 
diferenciadas de mortalidade entre esses dois tratamentos durante as diferentes fases de crescimento das mudas no viveiro. $\mathrm{O}$ tratamento convencional apresentou maior mortalidade durante a fase de crescimento após a saída das mudas da casa de sombra. O contrário foi constatado com a produção de mudas na espuma grande, cuja mortalidade está distribuída nas diferentes fases de produção (Figura 2). A mortalidade mais acentuada das mudas no tratamento com espuma grande pode estar relacionada à retenção de água, eventualmente em decorrência da secagem da parte superior da espuma dentro da casa de vegetação que foi observada durante a fase inicial do experimento. Na fase inicial das mudas dentro da casa de vegetação a umidade do ar e do "substrato" são importantes para a formação do sistema radicular. Essa secagem observada na parte superior de apenas algumas das espumas de maior comprimento sugere que a irrigação deve ser um dos principais pontos a serem acompanhados na possível adoção da espuma ou de produtos semelhantes para a produção de mudas de eucalipto clonal.

No experimento 2, observou-se que o tratamento convencional foi o que apresentou menor mortalidade, sendo que a mortalidade das mudas produzidas nos tratamentos com as espumas não apresentaram diferenças significativas no momento da expedição para o campo (Figura 3). Provavelmente, esse resultado pode ser reflexo do excesso de água que foi detectado durante o período da casa de vegetação.
O menor valor obtido (tratamento convencional) foi superior à mortalidade comumente encontrada nos viveiros estudados de acordo com os técnicos responsáveis. Esse fato pode representar a maior susceptibilidade das mudas produzidas na espuma ao excesso de água em relação às mudas produzidas no substrato convencional. Aliado a isso, também indica a necessidade de modificação da frequência ou da quantidade de água utilizada na irrigação com a utilização da espuma para produção das mudas, pois existe uma relação direta entre o substrato utilizado e a irrigação com a mortalidade das mudas de eucalipto no viveiro (LOPES et al., 2005).

\subsection{Produção de biomassa pelas mudas na fase de expedição do viveiro}

O maior valor absoluto de biomassa aérea do tratamento foi constatada com a espuma grande no experimento 1 , contudo, não tendo sido observadas diferenças significativas entre os tratamentos com espuma (Figura 4). A diferença significativa ocorreu apenas entre a espuma grande e o sistema convencional. No experimento 2, não foram constatadas diferenças significativas entre os tratamentos (Figura 5). Com referência à biomassa radicular, nenhuma diferença significativa foi encontrada entre os quatro tratamentos, em ambos os experimentos.

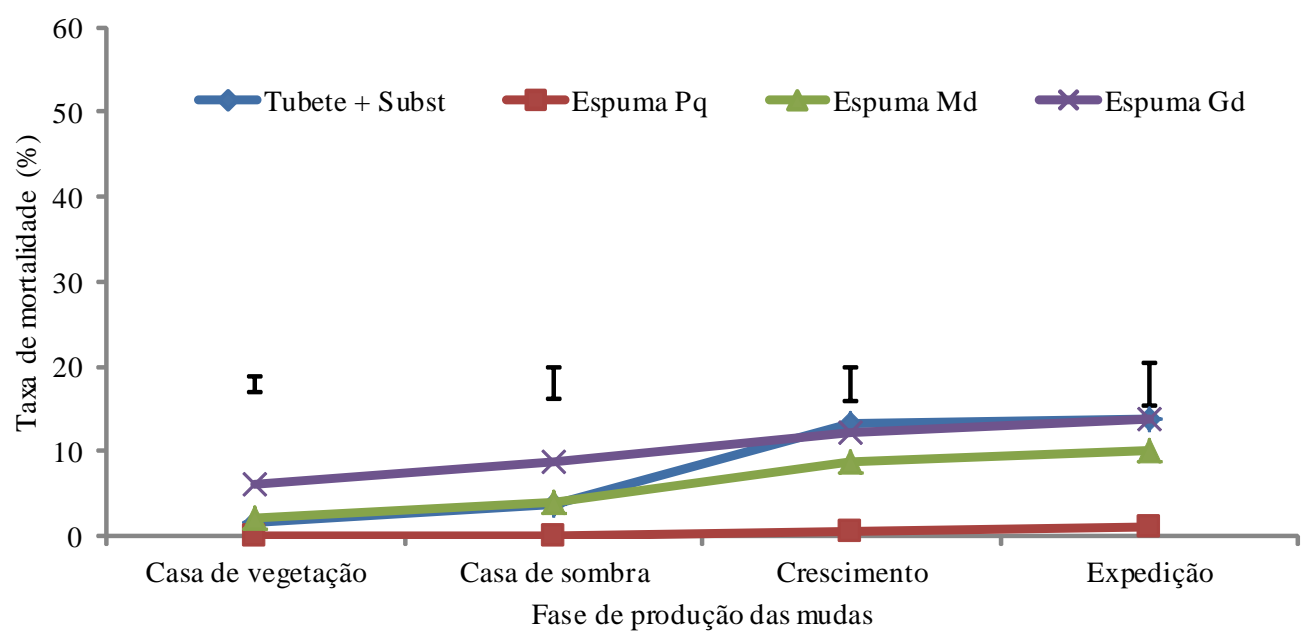

Figura 2 - Mortalidade das mudas em diferentes fases do experimento 1 (período de janeiro a abril de 2008) - As barras representam a diferença mínima significativa (Tukey - 0,05).

Figure 2 - Minicuttlings mortality at different stages in the experiment 1 (period: January to April) - The bars represent the least significant difference (Tukey - 0.05). 


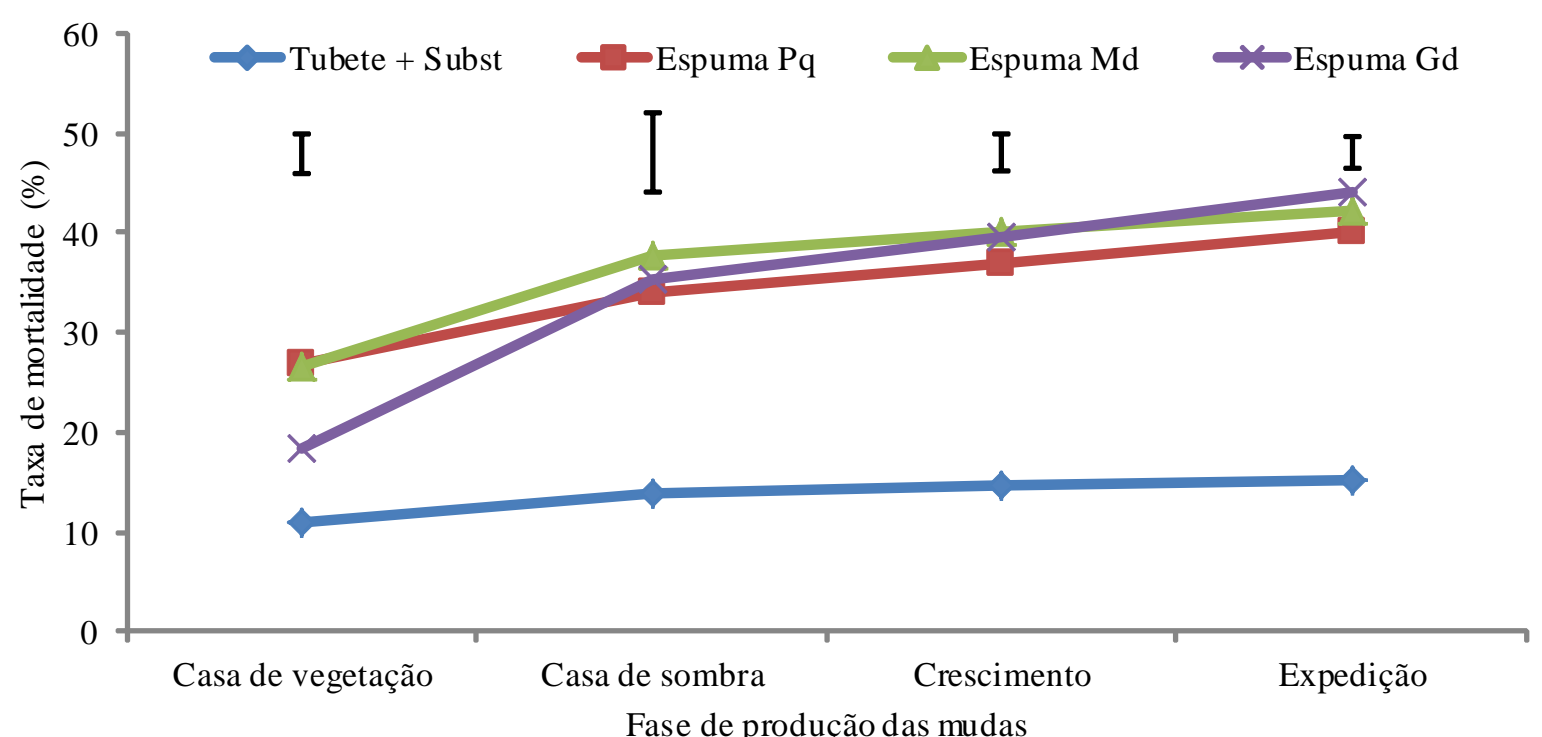

Figura 3 - Mortalidade das mudas em diferentes fases do experimento 2 (período de produção de maio a agosto de 2008) - As barras representam a diferença mínima significativa (Tukey - 0,05).

Figure 3-Minicuttings mortality at different stages in the second experiment (period: May to August) - The bars represent the least significant difference (Tukey - 0.05).

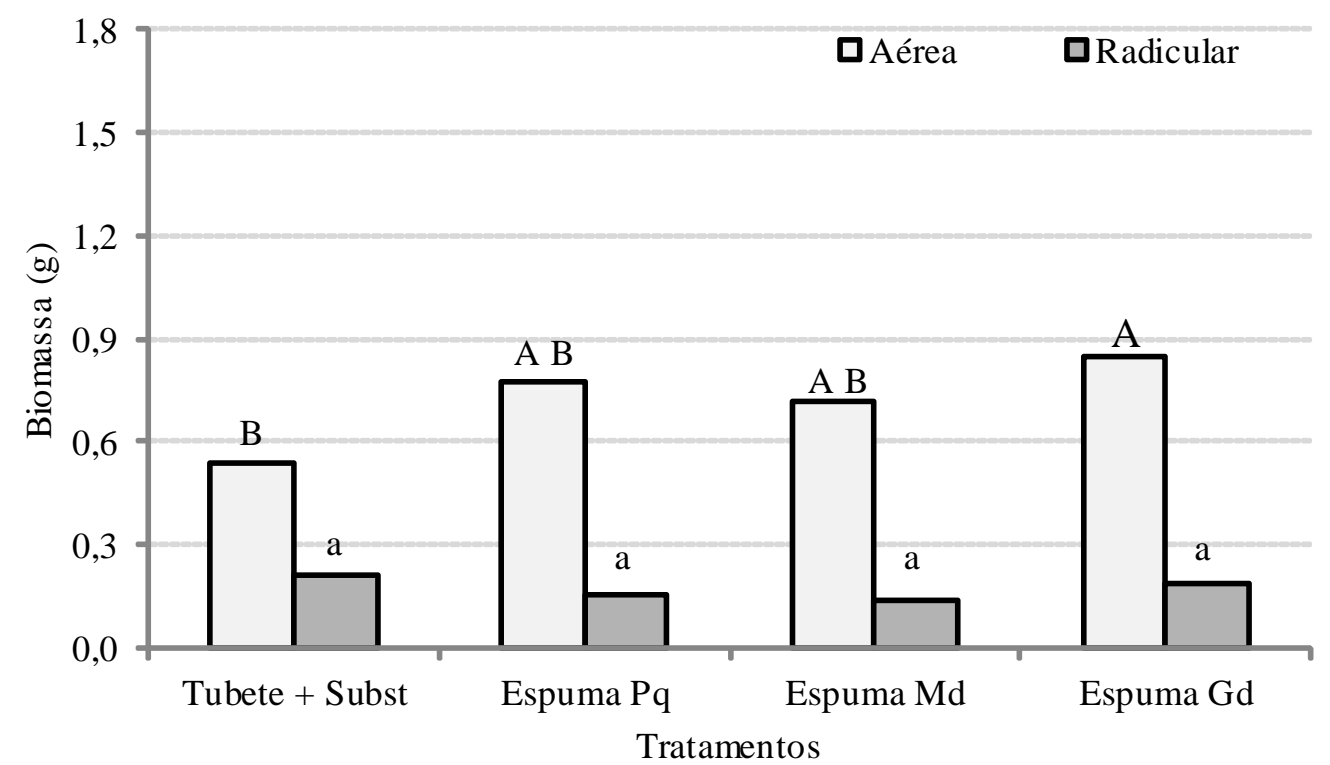

Figura 4 - Biomassas aérea e radicular na fase de expedição das mudas no experimento 1 (período: janeiro a abril) - Letras maiusculas e minusculas diferentes representam diferença estatistica da parte aérea e do sistema radicular, respectivamente entre os tratamentos pelo teste de Tukey $(\mathrm{P} \leq 0,05)$.

Figure 4-Shoot and root biomasses during the expedition in the first experiment (period: January to April) - Upper and lowercase letters represent different statistical difference of shoot and root, respectively, between treatments by Tukey test $(P \leq 0.05)$.

Cerne, Lavras, v. 18, n. 4, p. 639-649, out./dez. 2012 


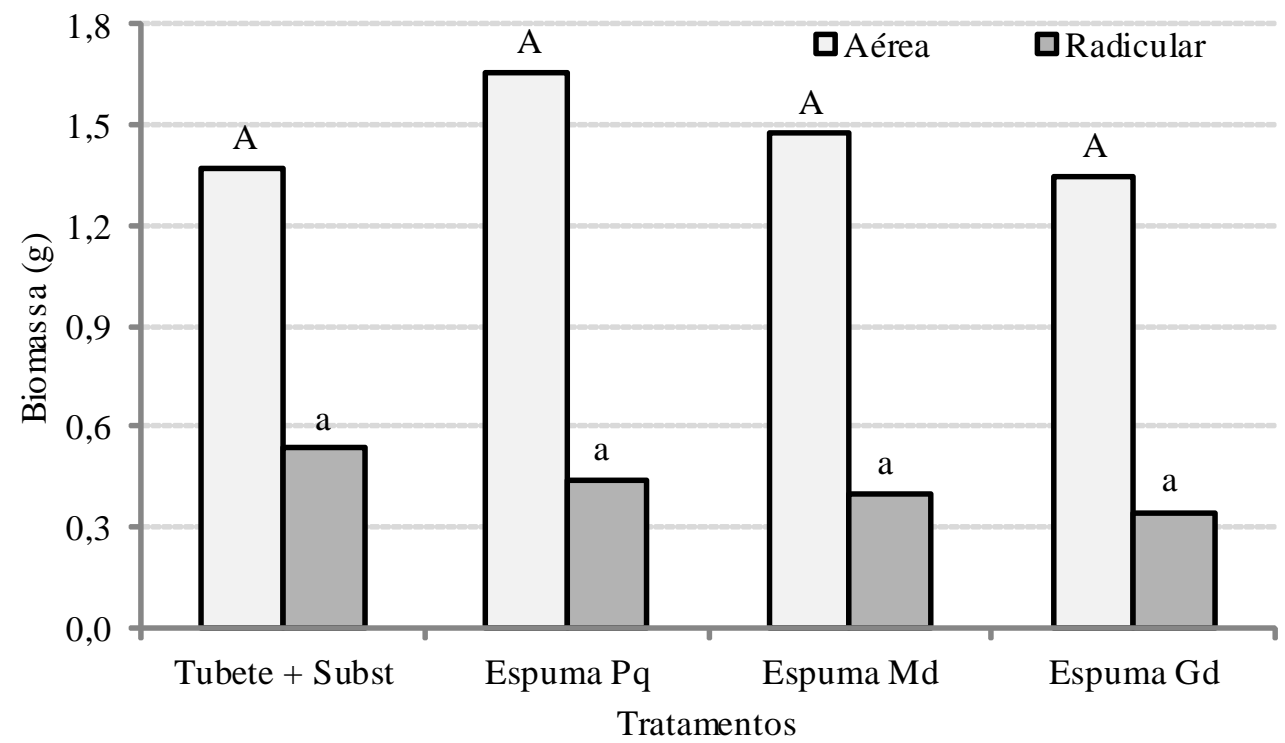

Figura 5 - Biomassas aérea e radicular na fase de expedição das mudas no experimento 2 (período: maio a agosto) - Letras maiusculas e minusculas diferentes representam diferença estatistica da parte aérea e do sistema radicular, respectivamente entre os tratamentos pelo teste de Tukey $(\mathrm{P} \leq 0,05)$.

Figure 5 - Shoot and root biomasses during the expedition in the second experiment (period: May to August) - Upper and lowercase letters represent different statistical difference of shoot and root, respectively, between treatments by Tukey test $(P \leq 0.05)$.

A biomassa aérea $\left(720 \mathrm{mg}\right.$ planta $\left.^{-1}\right)$ e radicular $(170$ $\left.\mathrm{mg}_{\text {planta }} \mathrm{p}^{-1}\right)$ média das mudas produzidas no experimento 1 foi inferior a biomassa aérea $\left(1.460 \mathrm{mg} \mathrm{planta}^{-1}\right)$ e radicular (430 $\mathrm{mg} \mathrm{planta}^{-1}$ ) do experimento 2 que obteve valores similares aos apresentados na literatura para expedição das mudas de eucalipto para o campo (MAFIA et al., 2005).

Sabe-se que modificações do substrato e da quantidade de água disponível para a planta durante a fase de produção das mudas alteram o crescimento e as características da parte aérea e do sistema radicular das mudas de eucalipto na fase de expedição (BARROSO et al., 2000; LOPES et al., 2007). Destaca-se que a espuma absorve e perde água por toda a superfície e esse fato deve gerar modificações na irrigação cujo resultado deverá ser trabalhado individualmente para as diversas fases de produção das mudas dentro dos viveiros.

A relação entre a biomassa da parte aérea e do sistema radicular das mudas foi diferente entre o tratamento convencional e os tratamentos com a utilização da espuma para produção das mudas, sendo a relação superior nos tratamentos com a espuma, em ambos os experimentos. No experimento 1, a relação obtida com a espuma média foi o dobro da relação obtida pelo tratamento convecional que foi de 2,6. No experimento 2 , o tratamento convecional obteve também uma relação de 2,6 que foi em média $30 \%$ inferior aos tratamentos com a produção de mudas nas espumas (Tabela 3).

Tabela 3 - Relação entre a biomassa área e radicular das mudas na fase de expedição em ambos os experimentos.

Table 3 - Relationship between shoot and root biomasses during the expedition to the field in both experimets.

\begin{tabular}{lrrrr}
\hline \multirow{2}{*}{ Tratamentos } & \multicolumn{4}{c}{ Experimento } \\
\cline { 2 - 6 } & \multicolumn{3}{c}{ Um } & \multicolumn{3}{c}{ Dois } \\
\hline Tubete + Substrato & 2,6 & $\mathrm{~b}$ & 2,6 & $\mathrm{~b}$ \\
Espuma Pq & 4,9 & $\mathrm{a}$ & 3,7 & $\mathrm{a}$ \\
Espuma Md & 5,2 & $\mathrm{a}$ & 3,7 & $\mathrm{a}$ \\
Espuma Gd & 4,5 & $\mathrm{a}$ & 3,9 & $\mathrm{a}$ \\
\hline
\end{tabular}

Letras diferentes na mesma coluna representam diferença estatistica entre os tratamentos pelo teste de Tukey $(P \leq 0,05)$.

\subsection{Mortalidade das mudas nos experimentos de campo}

No acompanhamento do crescimento inicial das mudas no campo, realizado em abril e maio no experimento 3 , até 30 dias do plantio, não foram observadas mudas

Cerne, Lavras, v. 18, n. 4, p. 639-649, out./dez. 2012 
mortas em nenhum dos tratamentos. No experimento 4, implantado em setembro, início da primavera, foram encontradas diversas mudas mortas, principalmente por falta de água. Mesmo com a aplicação do gel hidratado, foram observados $25 \%$ de mortalidade com as mudas provenientes de tubete e substrato após 30 dias do plantio. Para as mudas provenientes da espuma, os valores foram inferiores, sendo observados $10 \%$ para a espuma pequena e 3\% para as espumas médias e grandes. Esse resultado pode apresentar vantagem das mudas produzidas na espuma que teoricamente teriam maior reserva de água em relação às mudas produzidas no sistema convencional, principalmente, nas espumas com volume superior a 75 $\mathrm{cm}^{3}$. Destaca-se que a utilização de recipientes com maior volume gera vantagens ao crescimento das mudas, mas essas vantagens podem não ser suficientes para a adoção dos recipientes no sistema produtivo, em razão dos aspectos operacionais envolvidos (GOMES et al., 2003).

São esperadas respostas diferenciadas entre os materiais geneticos em relação à disponibilidade de água (TATAGIBA et al., 2007). Neste estudo, a diferença de mortalidade entre os experimentos 3 e 4 não se deve ao material genético, mas às condições de ambiente, pois no experimento 3 foram realizadas duas irrigações e as condições climáticas eram mais amenas (outono). No experimento 4, o plantio foi realizado com o gel hidratado e não foram realizadas irrigações após o plantio.
A principal causa de mortalidade das mudas de eucaliptos no campo é o déficit hídrico, mas também são comuns problemas com formigas cortadeiras e cupins (LOPES et al., 2009; VIERO; LITTLE, 2006). Aparentemente, as mudas produzidas com a utilização da espuma fenólica apresentaram pequena vantagem na tolerância ao déficit hídrico, mas em relação às pragas não foram observadas diferenças no campo.

\subsection{Biomassa produzida pelas mudas no campo}

Na avaliação da biomassa produzida, das partes aérea e radicular, aos três meses após o plantio, não foram observadas diferenças entre os tratamentos no experimento 3 (Figura 6). No experimento 4, foi observada diferença entre a biomassa aérea das mudas produzidas com utilização do tubete e do substrato com as mudas produzidas com a espuma de maior volume. Os tratamentos relacionados às espumas de dimensões pequena e média equivaleram-se ao do tubete, assim como ao tratamento em que se utilizou a espuma grande (Figura 7). O maior crescimento das mudas produzidas com a espuma grande pode estar relacionado ao menor estresse hídrico sofrido pelas mudas desse tratamento na fase pós-plantio, considerando que a maior disponibilidade de água melhora a eficiência na absorção dos nutrientes do solo pelas mudas de eucalipto(ORIKIRIZA et al., 2009; TATAGIBA et al., 2009).

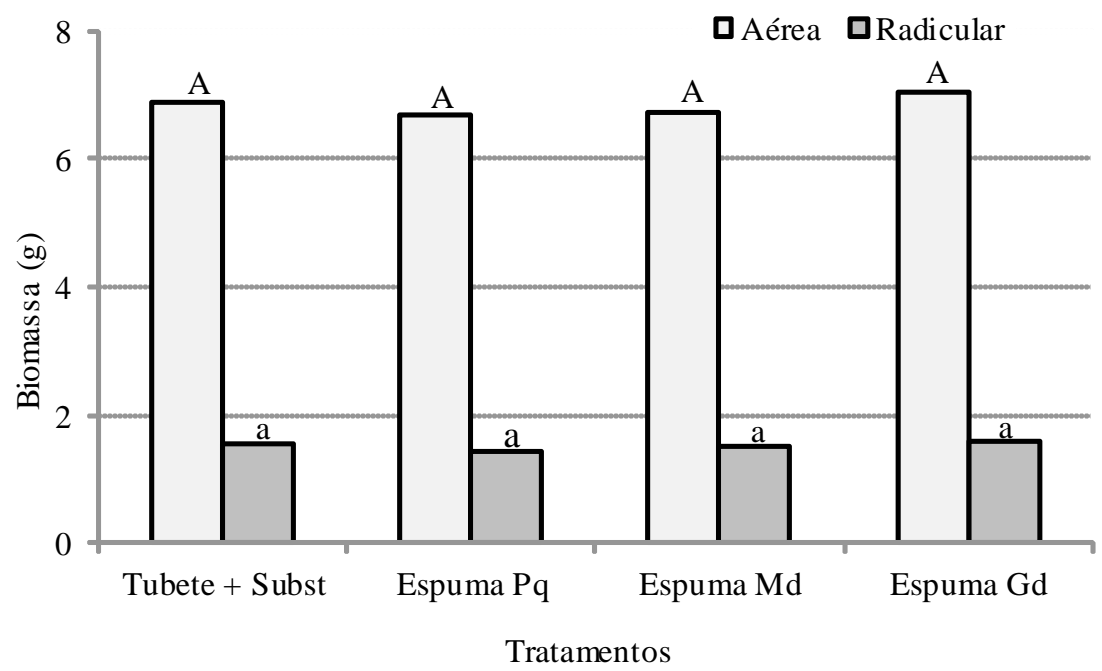

Figura 6 - Biomassa aérea e radicular das mudas, aos 3 meses de idade no campo, do experimento 3 (período: abril a julho) - Letras maiusculas e minusculas diferentes representam diferença estatistica da parte aérea e do sistema radicular, respectivamente entre os tratamentos pelo teste de Tukey $(\mathrm{P} \leq 0,05)$.

Figure 6 - Shoot and root biomass at 3 months in the field of the experiment 3 (period: April to July) - Upper and lowercase letters represent different statistical difference of shoot and root, respectively, between treatments by Tukey test $(P \leq 0.05)$.

Cerne, Lavras, v. 18, n. 4, p. 639-649, out./dez. 2012 


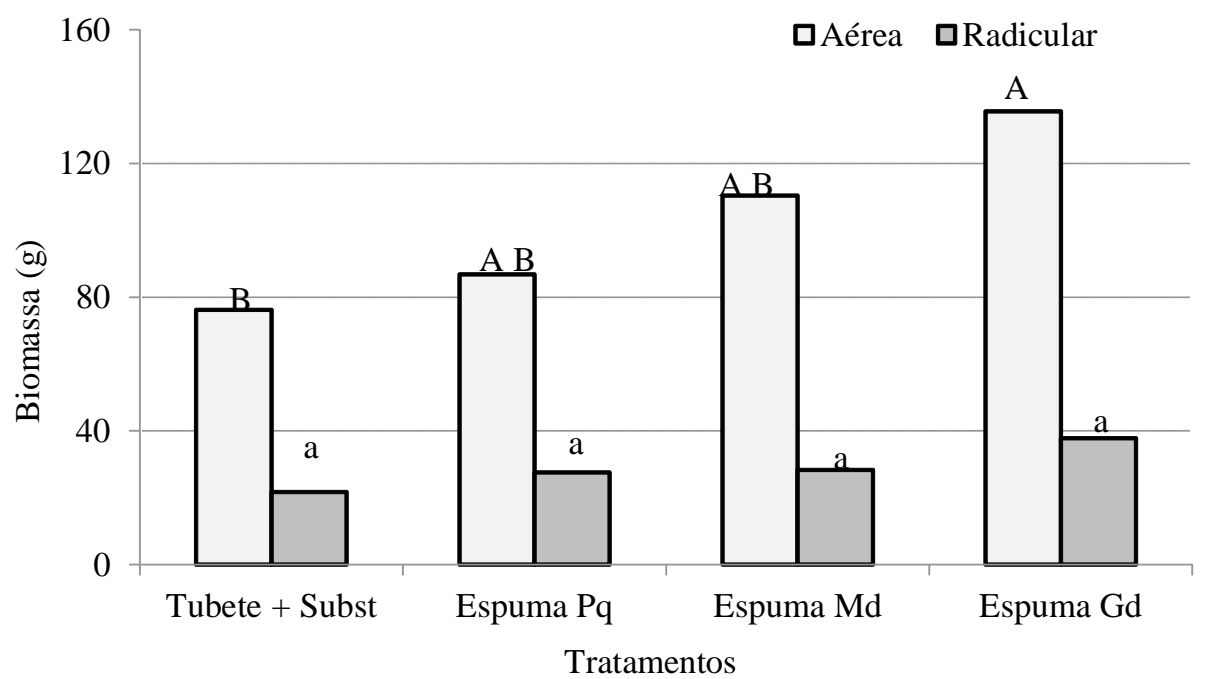

Figura 7 - Biomassa aérea e radicular das mudas, aos 3 meses de idade no campo, do experimento 4 (período: setembro a dezembro) Letras maiusculas e minusculas diferentes representam diferença estatistica da parte aérea e do sistema radicular, respectivamente entre os tratamentos pelo teste de Tukey $(\mathrm{P} \leq 0,05)$.

Figure 7-Shoot and root biomass at 3 months in the field in the experiment 4 (period: September to December) - Upper and lowercase letters represent different statistical difference of shoot and root, respectively, between treatments by Tukey test $(P \leq 0.05)$.

Em ambos os experimentos, aos três meses após o plantio no campo, não foram observadas diferenças na relação entre a biomassa produzida na parte aérea e a biomassa produzida no sistema radicular entre os tratamentos (Tabela 4). A diferença observada na relação entre a biomassa aérea e a biomassa do sistema radicular na fase de expedição das mudas do viveiro desapareceu rapidamente no campo. Esse resultado é importante pois o recipiente pode ter influência no crescimento das mudas no campo (CLOSE et al., 2006). As mudas de eucalipto, formadas com qualidade, não apresentam diferenças no crescimento, após alguns meses do plantio no campo, mesmo sendo implantadas no campo com menor biomassa radicular ou com diferenças morfológicas no sistema radicular geradas pelos diferentes tratamentos aplicados no viveiro (FERNÁNDEZ et al., 2007; FREITAS et al., 2009).

Mudanças no recipiente e/ou substrato de produção de mudas podem causar alterações significativas no processo de produção de mudas, como o manejo da irrigação e fertilização. Neste trabalho não foram feitas adaptações na irrigação ou na fertilização o que permitiu verificar que a principal adaptação do manejo está relacionada a irrigação no viveiro. Para a adoção das espumas ou de outros recipientes que tenham capacidade de absorção ou perda de água diferente dos tubetes
Tabela 4 - Relação entre a biomassa área e radicular das mudas após 3 meses do plantio no campo em ambos os experimentos de campo.

Table 4 - Relationship between shoot and root biomasses at 3 months in the field in both experimets.

\begin{tabular}{ccccc}
\hline \multirow{2}{*}{ Tratamentos } & \multicolumn{4}{c}{ Experimento } \\
\cline { 2 - 5 } & \multicolumn{3}{c}{ Três } & \multicolumn{2}{c}{ Quatro } \\
\hline Tubete + Substrato & 4,4 & $\mathrm{a}$ & 3,5 & $\mathrm{a}$ \\
Espuma Pq & 4,6 & $\mathrm{a}$ & 3,1 & $\mathrm{a}$ \\
Espuma Md & 4,5 & $\mathrm{a}$ & 3,9 & $\mathrm{a}$ \\
Espuma Gd & 4,4 & $\mathrm{a}$ & 3,6 & $\mathrm{a}$ \\
\hline
\end{tabular}

convecionais, a mudança na frequência e na lamina d'água deve ser estudada conforme Ferraz e Cereda (2010). Possivelmente a utilização de recipientes cuja superficie absorve e perde água será necessario um aumento na frequência, mas com menor quantidade de água. No campo não foram observadas diferenças significativas entre os sistemas estudados.

\section{CONCLUSÕES}

A substituição do sistema convencional com a utilização de tubete e do substrato para a produção de

Cerne, Lavras, v. 18, n. 4, p. 639-649, out./dez. 2012 
mudas clonais de eucalipto pelo sistema que utiliza a espuma é viável do ponto de vista biológico. No entanto, para a adoção desse sistema será necessário realizar adaptações no viveiro, principalmente, na irrigação.

\section{AGRADECIMENTOS}

Agradecemos aos colegas Israel Gomes Vieira, Marcela Trecenti Capoani, Yara Mosca, Karina Lima, Rildo Moreira Moreira, Donizete Aparecido Sabino, aos acadêmicos Letícia Mantovani Stein e Rômulo Barbiéri que auxiliaram na execução do projeto, ao Departamento de Ciências Florestais da ESALQ/USP e à empresa Lwarcel pelo apoio.

\section{REFERÊNCIAS}

ASSOCIAÇÃO BRASILEIRA DE PRODUTOS DE FLORESTAS PLANTADAS. Anuário estatístico da ABRAF 2009: ano base 2008. Brasília, 2009. Disponível em: <http://www.ipef.br/estatisticas/relatorios/anuario-ABRAF2009.pdf>. Acesso em: 10 out. 2011.

BARROSO, D. G.; CARNEIRO, J. G. A.; LELES, P. S. S.; MORGADO, I. F. Regeneração de raízes de mudas de eucalipto em recipientes e substratos. Scientia Agricola, Piracicaba, v. 57, n. 2, p. 229-237, 2000.

CLOSE, D. C.; BAIL, I.; HUNTER, S.; BEADLE, C. L. Defining seedling specifications for Eucalyptus globulus: effects of seedling size and container type on early afterplanting performance. Australian Forestry, Queen Victoria, v. 69 , n. 1, p. $2-8,2006$.

FERNANDES, A. A.; MARTINEZ, H. E. P.; SILVA, D. J. H.; BARBOSA, J. G. Produção de mudas de tomateiro por meio de estacas enraizadas em hidroponia. Pesquisa Agropecuária Brasileira, Brasília, v. 39, n. 4, p. 533-537, abr. 2004.

FERNÁNDEZ, M.; TEJERO, J. R.; PÉREZ, I.; SORIA, F.; RUIZ, F.; LÓPEZ, G. Effect of copper coating nursery containers on plant growth and root morphology of Eucalyptus globulus Labill. cuttings and seedlings. Silva Lusitana, Oeiras, v. 15, n. 2, p. 215-227, 2007.

FERRAZ, M. V.; CEREDA, M. P. Determinação das características morfológica de petúnias comuns (petunia $\mathrm{x}$ hybrida) cultivadas em tubetes biodegradáveis. Scientia Agraria Paranaensis, Cascavel, v. 9, n. 1, p. 95-107, 2010 .

Cerne, Lavras, v. 18, n. 4, p. 639-649, out./dez. 2012
FREITAS, T. A. S.; BARROSO, D. G.; CARNEIRO, J. G. A.; PENCHEL, R. M.; LAMÔNICA, K. R.; FERREIRA, D. A. Desempenho radicular de mudas de eucalipto produzidas em diferentes recipientes e substratos. Revista Árvore, Viçosa, v. 29, n. 6, p. 853-861, nov./dez. 2005.

FREITAS, T. A. S.; BARROSO, D. G.; SOUZA, L. S.; CARNEIRO, J. G. A. Efeito da poda de raizes sobre o crescimento das mudas de eucalipto. Ciência Florestal, Santa Maria, v. 19, n. 1, p. 1-6, 2009.

GOMES, J. M.; COUTO, L.; LEITE, H. G.; XAVIER, A.; GARCIA, L. S. R. Crescimento de mudas de Eucalyptus grandis em diferentes tamanhos de tubetes e fertilização N-P-K. Revista Árvore, Viçosa, v. 27, n. 2, p. 113-127, 2003.

LINDGREN, D. Advantages of clonal propagation. In: WORKSHOP ON HIGH QUALITY BIRCH: CLONAL PROPAGATION AND WOOD PROPERTIES, 2002, Ronneby. Proceedings... Ronneby, 2002. p. 98-109.

LOPES, J. L. W.; GUERRINI, I. A.; SAAD, J. C. C. Qualidade de mudas de eucalipto produzidas sob diferentes lâminas de irrigação e dois tipos de substrato. Revista Árvore, Viçosa, v. 31, n. 5, p. 835-843, set./out. 2007.

LOPES, J. L. W.; GUERRINI, I. A.; SAAD, J. C. C.; SILVA, M. R. Efeitos da irrigação na sobrevivência, transpiração e no teor relativo de água na folha em mudas de Eucalyptus grandis em diferentes substratos. Scientia Florestalis, Piracicaba, n. 68 , p. 97-106, 2005.

LOPES, J. L. W.; SAAD, J. C. C.; GUERRINI, I. A.; LOPES, C. F. Influência dos fatores bióticos e abióticos na sobrevivência de eucalipto em função do solo e do manejo de viveiro. Biotemas, Florianópolis, v. 22, n. 2, p. 29-38, 2009.

MAFIA, R. G.; ALFENAS, A. C.; SIQUEIRA, L.; FERREIRA, E. M.; LEITE, H. G.; CAVALLAZZI, J. R. P. Critério técnico para determinação da idade ótima de mudas de eucalipto para plantio. Revista Árvore, Viçosa, v. 29, n. 6, p. 947-953, 2005.

MATIAS, G. C. S.; COMETTI, N. N.; GÓMEZ, G. P.; ROCHA, J. D. S. Avaliação de substratos comerciais para a produção de mudas de alface. Horticultura Brasileira, Brasília, v. 17, n. 2, p. 159-162, 1999. 
MCNABB, K.; GONÇALVES, N.; GONÇALVES, J. Clonal propagation of Eucalyptus in Brazilian nurseries. In: UNITED STATES DEPARTMENT OF AGRICULTURE. Rocky mountain research station. Washington, 2002. p. 165-168. (Forest Service, RMRS-P24).

ORIKIRIZA, L. J. B.; AGABA, H.; TWEHEYO, M.; EILU, G.; KABASA, J. D.; HÜTTERMANN, A. Amending soils with hydrogels increases the biomass of nine tree species under non-water stress conditions. CLEAN - Soil, Air, Water, London, v. 37, n. 8, p. 615$620,2009$.

PAULUS, D.; MEDEIROS, S. L. P.; SANTOS, O. S.; RIFFEL, C.; FABRIN, E. G.; PAULUS, E. Substratos na produção hidropônica de mudas de hortelã. Horticultura Brasileira, Brasília, v. 23, n. 1, p. 48-50, 2005.

PRIETO-RUIZ, J. A.; DOMÍNGUEZ-CALLEROS, P. A.; CORNEJO-OVIEDO, E. H.; NÁVAR-CHÁIDEZ, J. J. Efecto del envase y del riego en vivero en El establecimiento de Pinus cooperi Blanco em dos condiciones de sitio. Madera y Bosques, Xalapa, v. 13, n. 1, p. 79-97, 2007.

STAPE, J. L.; GONÇALVES, J. L. M.; GONÇALVES, A. N. Relationships between nursery practices and field performance for Eucalyptus plantations in Brazil: a historical overview and its increasing importance. New Forests, Dordrecht, v. 22, n. 1/ 2, p. 19-24, 2001.

TATAGIBA, S. D.; PEZZOPANE, J. E. M.; REIS, E. F. dos. Avaliação do crescimento e produção de clones de Eucalyptus submetidos a diferentes manejos de irrigação. Cerne, Lavras, v. 13, n. 1, p. 1-9, 2007.

TATAGIBA, S. D.; PEZZOPANE, J. E. M.; REIS, E. F. dos; PENCHEL, R. M. Desempenho de clones de eucalipto em resposta a disponibilidade de água no substrato. Engenharia na Agricultura, Viçosa, v. 17, n. 3, p. 179-189, 2009.

VIERO, P.; LITTLE, K. Results from a two year Eucalyptus smithii re-establishment trial situated in the KwaZulu-Natal Midlands, South Africa. London: ICFR, 2006. 10 p. (Bulletin Series, 14). 